\title{
LOCALIZACIÓN DEL FINAL DEL NEOGLACIAR A PARTIR DEL GRADIENTE GEOTÉRMICO MEDIDO EN EL SONDEO DE VITORIA DE 1880
}

\author{
Artemio Cuenca Payá
}

\section{INTRODUCCIÓN}

Box Amorós (1985), para explicar ciertos fenómenos relacionados con la Laguna de Salinas (Alicante) postula un posible cambio climático entre los siglos XVII y XVIII justificándolo en el aumento anormal de las crecidas de los ríos Segura y Júcar a partir de aquellas fechas y que podría interpretarse como una variación en el régimen de precipitaciones.

La discusión de este punto indujo al autor a revisar algunos datos que pudiesen dilucidar la cuestión utilizando la metodología propuesta por Cermak (1971) y modificada por Cuenca Payá y Walker (1982), fundamentada en el estudio de las desviaciones del gradiente geotérmico medidas en sondeos profundos. El problema para aplicar este método era la ausencia de datos para España, pues rara vez se realizan esas mediciones en pozos libres de influencias extrañas (circulación de agua, focos geotérmicos, etc.), pero afortunadamente sí se hicieron en un pozo perforado en Vitoria entre los años 1877 y 1881 (Anónimo, 1929:17) del que son utilizables las medidas tomadas a 100, 200, 300 y 400 metros de profundidad (Cuadro I) permitiendo las restantes, hasta 800 metros, calcular la recta de regresión del gradiente, teniendo ésta por ecuación

$$
\mathrm{T}=9,5900+0,0308 \mathrm{z}
$$

con un coeficiente de correlación

$$
\mathrm{r}=0,999984
$$

siendo $\mathrm{T}$ la temperatura en ${ }^{\circ} \mathrm{C} \mathrm{y}$ «z» la profundidad en metros. 


\section{CUADRO I}

\begin{tabular}{ccc}
$\frac{Z}{100}$ & $\frac{T}{2}$ & $\begin{array}{c}\text { Desviación del } \\
\text { gradiente }\end{array}$ \\
\cline { 3 - 3 } 200 & 14,25 & 1,58 \\
300 & 17,10 & 1,35 \\
400 & 19,50 & 0,67 \\
& 22,50 & 0,59
\end{tabular}

Con sólo cuatro lecturas fuera del gradiente poco puede inferirse en cuanto a variaciones climáticas pasadas, pero como el problema se centraba en determinar un solo cambio, una sencilla combinación de las metodologías propuestas por los autores antes citados podía permitir detectar el momento de la ruptura siempre y cuando se «laminaran» los picos y se considerasen dos niveles de temperaturas constantes separadas por un salto en escalón.

\section{METODOLOGÍA}

Se fundamenta en el problema clásico de propagación del calor en régimen transitorio hacia el interior de un semiespacio y desde su superficie. El caso aquí estudiado sería el de determinar dos diferentes temperaturas $\mathrm{T}_{1} \mathrm{y}_{2}$ mantenida la primera un cierto tiempo desde 1880 hacia el pasado y seguida después por la $T_{2}$ durante otro período también desconocido; la desviación en el gradiente geotérmico $\Delta \mathrm{T}(\mathrm{z})$ inducido por $\mathrm{T}_{1}$ a una profundidad $\mathrm{z}$ vendrá dada por

$$
>\mathrm{T}_{1}(\mathrm{z})=\mathrm{T}_{1}\left[\operatorname{erf}\left(\mathrm{z}, \mathrm{t}_{1}\right)-\operatorname{erf}\left(\mathrm{z}, \mathrm{t}_{2}\right)\right]
$$

y lo mismo puede plantearse para $\mathrm{T}_{2}$.

Cermak utiliza la derivada de esta ecuación para operar con gradientes, pero Cuenca Payá y Walker proponen calcular las T directamente de (2), para lo cual es necesario multiplicar los $\Delta \mathrm{T}(\mathrm{z})$ por [1-erf $\left.\left(\mathrm{z}, \mathrm{t}_{\mathrm{n}}\right)\right]$, es decir, por la unidad menos el valor de la integral de Gauss obtenido para el último intervalo, al objeto de eliminar la «memoria» de temperaturas anteriores.

Puesto que se desconoce la duración de los intervalos de aplicación de las $\mathrm{T}$ deben ensayarse pares de intervalos distintos para cada par de desviaciones del gradiente realmente medidas, lo que en este caso, con cuatro mediciones, equivale a resolver seis sistemas de dos ecuaciones por cada ensayo. Lógicamente, el intervalo mejor ajustado será el que proporcione seis pares de valores para $\mathrm{T}_{1}$ y $\mathrm{T}_{2}$ con la mínima desviación standard para ambas, proponiendo Cermak la expresión: 


$$
\min \left(\mathrm{S}=\mathrm{s}_{1}^{2} \mathrm{~s}_{2}{ }_{2}\right)
$$

siendo $\mathrm{s}_{1} \mathrm{y} \mathrm{s}_{2}$ las desviaciones standard de las $\mathrm{T}_{1} \mathrm{y}_{2}$.

\section{RESULTADOS}

Se han ensayado cuatro pares de intervalos partiendo del año 1880 hacia atrás con límites situados en 1700-1280, 1650-1180, 1550-1100 y 1550-1280 indicando el primer año de cada intervalo el momento de cambio buscado, y el segundo el límite máximo hasta el que es posible extender las condiciones térmicas anteriores a dicho momento. Los resultados se exponen en el Cuadro 2 donde aparecen valores suficientemente bajos de $\mathrm{S}$ para los dos pares finales, presentando el último un mejor ajuste con $\Delta \mathrm{T}_{1}=1,88^{\circ} \mathrm{C}, \Delta \mathrm{T}_{2}=0,28^{\circ} \mathrm{C}$ y $\mathrm{S}=0,433$, lo que teniendo en cuenta la (1) se traduce en valores de la temperatura real de

$$
\begin{aligned}
& \mathrm{T}_{1}=11,47^{\circ} \mathrm{C} \\
& \mathrm{T}_{2}=9,87^{\circ} \mathrm{C}
\end{aligned}
$$

el primero extendido desde 1880 hasta mediados del siglo XVI y el segundo desde esas fechas hasta las postrimerías del siglo XIII.

\section{CUADRO II}

\begin{tabular}{llll}
\hline Límites de intervalos & $\frac{T 1}{2,8881}$ & $\frac{T 2}{0,638}$ & $\frac{S}{1,212}$ \\
$1880-1700-1280$ & 2,395 & 0,608 & 0,706 \\
$1880-1650-1180$ & 2,005 & 0,403 & 0,407 \\
$1880-1550-1100$ & 1,880 & 0,281 & 0,433 \\
$1880-1550-1280$ & & & \\
\hline
\end{tabular}

Es fácil localizar en ese segundo intervalo al Neoglaciar (Pequeña Edad del Hielo) coincidiendo bastante aproximadamente con los resultados obtenidos por Cuenca Payá y Walker para la Cuenca del Calumet (Illinois) aunque en Vitoria con una temperatura más alta, hecho que puede imputarse a las características más oceánicas del clima del Norte peninsular respecto a las fuertemente continentales de Illinois, o simplemente a la «laminación» de pico, producida al tener que trabajar con tan pocas medidas.

También es de señalar la coincidencia en el tiempo con los datos isotópicos obtenidos en el interior de Groenlandia (Gribbin y Lamb, 1979: 72) frente al desfase detectado en Islandia, Islas Británicas y costa NW europea donde el 
Neoglaciar se «retrasa» aproximadamente dos siglos y llega hasta ca. 1850. Posiblemente influya en esto la inercia de la Corriente del Golfo ante el cambio térmico, pero esa discusión ya escapa a los objetivos aquí buscados y merece por sí misma una investigación más detallada.

\section{CONCLUSIONES}

Como resumen de lo expuesto puede confirmarse que la Península Ibérica quedó sometida al descenso térmico del Neoglaciar en una época que coincide con la determinada para ese evento en otras regiones del globo, pero que es precoz respecto al Atlántico Norte y NW europeo posiblemente por condicionantes locales en estas zonas.

El ascenso de las temperaturas ya se registra hacia la mitad o finales del siglo XVI, elevándose hasta valores similares a los actuales, aunque esto debe contemplarse con suma prudencia pues los casi $12{ }^{\circ} \mathrm{C}$ de $\mathrm{T}_{1}$ son un promedio después de la «laminación» de posibles oscilaciones menores.

Respecto a las precipitaciones nada puede decirse, pero no deja de ser sintomático que cuando las temperaturas alcanzan su media actual tras el Neoglaciar, el registro de las crecidas del Júcar y del Segura se dispare espectacularmente y ambos ríos inicien su régimen de torrencialidad tan característico hoy día; por otra parte no hay razones para pensar que los cronistas del siglo XVI fuesen menos eficientes y responsables que sus colegas de los siglos XVII y XVIII en cuanto a dar fe de catástrofes con incidencia directa en la economía local. Pero dejando aparte especulaciones sobre la interpretación de lo registrado en los viejos archivos, se tiene la evidencia del paso del Neoglaciar a las condiciones actuales fundamentada en medidas físicas libres de cualquier subjetividad.

\section{BIBLIOGRAFÍA}

ANÓNIMO. 1929. «Los sondeos antiguos y los grandes sondeos europeos». Publicaciones de la Com. Nac. de Sondeos, t. 1, p. 17.

BOX AMORÓS, M.: 1985. Las zonas húmedas de la Provincia de Alicante y los procesos de intervención antrópica. Tesis de Doctorado, Fac. de Filosofía y Letras, Univ. de Alicante, en prensa.

CERMAK, V.: 1971. «Underground temperature and inferred climatic temperature of the past millenium». Paleogeography, Paleoclimatol., Paleoecol., 10, pp. 1-19.

CUENCA PAYÁ, A., y WALKER, M. J.: 1982. «Una evaluación de las temperaturas medias seculares durante el último milenio a partir de las desviaciones del gradiente geotérmico». Anal. Univ. de Alicante, Historia Medieval, 1, pp. 245-251.

GRIBBIN, J., y LAMB, H. H.: 1979. «Climatic change in historical times», en Gribbin, J. ed., Climatic change, pp. 68-82, Cambridge University Press. 